\title{
THE NUMBER OF SOLUTIONS TO LINEAR DIOPHANTINE EQUATIONS AND MULTIVARIATE SPLINES
}

\author{
WOLFGANG DAHMEN AND CHARLES A. MICCHELLI
}

\begin{abstract}
In this paper we study how the number of nonnegative integer solutions of $s$ integer linear equations in $n \geq s$ unknowns varies as a function of the inhomogeneous terms. Aside from deriving various recurrence relations for this function, we establish some of its detailed structural properties. In particular, we show that on certain subsets of lattice points it is a polynomial. The univariate case $(s=1)$ yields E. T. Bell's description of Sylvester's denumerants.

Our approach to this problem relies upon the use of polyhedral splines. As an example of this method we obtain results of $R$. Stanley on the problem of counting the number of magic squares.
\end{abstract}

1. Introduction. Let $X$ be an $s \times n$ integer matrix with columns $x^{1}, \ldots, x^{n} \in$ $\mathbf{Z}^{s} \backslash\{0\}$ such that the only solution of the equation $X \lambda=0, \lambda \in \mathbf{R}_{+}^{n}$ is the zero vector, i.e.,

$$
0 \notin\left[\left\{x^{1}, \ldots, x^{n}\right\}\right]
$$

where $[A]$ denotes the convex hull of a given set $A$. Consequently, if we denote the cardinality of $A$ by $|A|$ we see that

$$
t(\alpha \mid X)=\left|\left\{\beta \in \mathbf{Z}_{+}^{s}: X \beta=\alpha\right\}\right|
$$

is finite for every $\alpha \in \mathbf{Z}^{s}$.

The objective of this paper is to study $t(\cdot \mid X)$ as a function on $\mathbf{Z}^{s}$. For the case $s=1$, i.e., when we have a single linear relation, $t(\cdot \mid X)$ was introduced by Sylvester who called it a denumerant. It was further studied by E. T. Bell who proved that $t(\cdot \mid X)$ is a polynomial on certain subsets of the integers (cf. $[\mathbf{2}, \mathbf{1 2}])$. In general, $t(\cdot \mid X)$ is relevant for the combinatorial theory of partitions of nonnegative integers (see [11] for some special two dimensional examples) and in a special case it corresponds to the problem of counting matrices with nonnegative integer entries and equal row and column sums (magic squares). Recently, Stanley [13] used properties of Hilbert series of graded algebras to prove certain conjectures about magic squares stated in [1] (see $\S \S 2,4$ for more details). We will show that these results also appear as a consequence of Theorem 3.1 below.

Our interest in this subject arose from some recent work on the multivariate truncated power $T(\cdot \mid X)$ and box spline $B(\cdot \mid X)$. Under the assumption (1.1) they

Received by the editors November 7, 1986.

1980 Mathematics Subject Classification (1985 Revision). Primary 10B30, 10A21, 10A50, 05B35, 41A15, 41A63, 39A70.

This work was partially supported by NATO Grant No. DJ RG 639/84. 
are defined respectively by the equations

$$
\int_{\mathbf{R}^{s}} f(x) T(x \mid X) d x=\int_{\mathbf{R}_{+}^{n}} f(X u) d u,
$$

and

$$
\int_{\mathbf{R}^{s}} f(x) B(x \mid X) d x=\int_{[0,1]^{n}} f(X u) d u
$$

which are assumed to hold for any continuous function $f$ of compact support in $\mathbf{R}^{s}$. (Although these definitions do not require $X$ to have integer entries, we will only deal with this case here.) When $\operatorname{span}\left\{x^{1}, \ldots, x^{n}\right\}=\langle X\rangle=\mathbf{R}^{s}$, both $T(x \mid X)$ and $B(x \mid X)$ are piecewise polynomials of degree $\leq n-s$. We will assume in what follows that $\langle X\rangle=\mathbf{R}^{s}$. For more detailed information about these functions the reader is referred to $[\mathbf{3}, \mathbf{4}, \mathbf{6}]$. The identity

$$
T(x \mid X)=\sum_{\alpha \in \mathbf{Z}^{s}} t(\alpha \mid X) B(x-\alpha \mid X)
$$

established in [6] shows that these three functions are closely related. This equation proved useful in connection with subdivision algorithms for the fast computation of box spline surfaces [9]. In the present context, it will allow us to use multivariate splines to study $t(\cdot \mid X)$.

The paper is organized as follows: After deriving some basic identities for $t(\cdot \mid X)$ we motivate in $\S 2$ the subsequent discussion by recalling Bell's characterization of Sylvester's denumerant and relating $t(\cdot \mid X)$ to the problem of counting magic squares. The main result, Theorem 3.1, along with some consequences like the extension of Bell's theorem to systems of equations, is given in $\S 3$. The first part of Theorem 3.1 states that on certain cones depending on $X, t(\cdot \mid X)$ coincides with an element of the intersection of the null spaces of certain difference operators. This element is uniquely determined by certain interpolation conditions. The second part of Theorem 3.1 gives a reciprocity relation for these extensions of the enumerating function $t(\cdot \mid X)$ which, as pointed out before, is only well defined when (1.1) holds. We mention that when (1.1) is not required to hold similar reciprocity relations but for Hilbert series related to sets of solutions to linear diophantine equations are obtained in [14] by totally different methods. The results of $\S 3$ are then applied in $\S 4$ to the problem of counting magic squares. Combining Theorem 3.1 with the characterization of the above mentioned intersection of null spaces we begin $\S 5$ by proving our extension of Bell's theorem. Furthermore, in this section we exploit the relationship of $t(\cdot \mid X)$ to multivariate splines and establish more detailed information about $t(\cdot \mid X)$. Specifically, we determine its leading homogeneous terms. This close relationship to the splines also gives a direct proof of the first part of Theorem 3.1 when $X$ is unimodular. Finally, $\S 6$ is devoted to proving Theorem 3.1 in its full generality.

2. Background material and some basic identities. The relation between $t(\cdot \mid X)$ and the multivariate truncated power becomes apparent if we define $t(\cdot \mid X)$ alternatively by requiring that the equation

$$
\sum_{\alpha \in \mathbf{Z}^{s}} \psi(\alpha) t(\alpha \mid X)=\sum_{\beta \in \mathbf{Z}_{+}^{n}} \psi(X \beta)
$$


holds for any $\psi: \mathbf{Z}^{s} \rightarrow \mathbf{R}$ which vanishes on all but a finite number of lattice points. This observation motivated us to call $t(\cdot \mid X)$ the discrete truncated power. Using (2.1), formula (1.5) readily follows from (1.3), (1.4) by decomposing $\mathbf{R}_{+}^{n}$ into integer translates of the cube $[0,1]^{n}$. The relation (2.1) also suggests the following: for any integer diagonal matrix $D=\operatorname{diag}\left(d_{1}, \ldots, d_{n}\right)$, set $d=\left(d_{1}, \ldots, d_{n}\right)$ and

$$
\sum_{\alpha \in \mathbf{Z}^{s}} \psi(\alpha) b_{D}(\alpha \mid X)=\sum_{\substack{\beta \in \mathbf{Z}_{+}^{n} \\ 0 \leq \beta<d}} \psi(X \beta) .
$$

Comparing this equation with (1.4) we call $b_{D}(\alpha \mid X), \alpha \in \mathbf{Z}^{s}$, the discrete box spline. Equivalently, (2.2) means

$$
b_{D}(\alpha \mid X)=\left|\left\{\beta \in \mathbf{Z}_{+}^{n}: X \beta=\alpha, 0 \leq \beta_{i}<d_{i}, i=1, \ldots, n\right\}\right|
$$

so that the discrete box spline counts the number of integer solutions of $X \beta=\alpha$ in the open cube $\left[0, d_{1}\right) \times \cdots \times\left[0, d_{n}\right)$. Formally, we have $t(\cdot \mid X)=b_{\infty}(\cdot \mid X)$.

Let us introduce the backward difference operator

$$
\nabla_{y} f(\alpha)=f(\alpha)-f(\alpha-y) .
$$

For notational convenience we will occasionally view $X$ as an (indexed) set of not necessarily distinct vectors $x^{1}, \ldots, x^{n}$ rather than as a matrix. Therefore, we can define inductively for $y \in X$

$$
\nabla_{X} f=\nabla_{y}\left(\nabla_{X \backslash\{y\}} f\right) .
$$

The discrete box spline $b_{D}(\alpha \mid X)$ is then related to $t(\alpha \mid X)$ by the equation

$$
b_{D}(\alpha \mid X)=\nabla_{X D} t(\alpha \mid X)
$$

which is the discrete analog of the relation

$$
B(x \mid X)=\nabla_{X} T(x \mid X),
$$

[6]. For a discrete analog to (1.5), we observe that

$$
\begin{aligned}
\sum_{\alpha \in \mathbf{Z}^{s}} \psi(\alpha) t(\alpha \mid X) & =\sum_{\beta \in \mathbf{Z}_{+}^{n}} \psi(X \beta)=\sum_{\mu \in \mathbf{Z}_{+}^{n}} \sum_{0 \leq \beta<d} \psi(X D \mu+X \beta) \\
& =\sum_{0 \leq \beta<d} \sum_{\alpha \in \mathbf{Z}^{s}} \psi(\alpha+X \beta) t(\alpha \mid X D) \\
& =\sum_{\alpha \in \mathbf{Z}^{s}} \sum_{\gamma \in \mathbf{Z}^{s}} \psi(\alpha+\gamma) b_{D}(\gamma \mid X) t(\alpha \mid X D) \\
& =\sum_{\alpha \in \mathbf{Z}^{s}} \psi(\alpha) \sum_{\nu \in \mathbf{Z}^{s}} t(\nu \mid X D) b_{D}(\alpha-\nu \mid X)
\end{aligned}
$$

which means

$$
t(\alpha \mid X)=\sum_{\nu \in \mathbf{Z}^{s}} t(\nu \mid X D) b_{D}(\alpha-\nu \mid X)
$$

This equation relates partitions with respect to $X, X D$ and restricted partitions for $X$. 
Replacing $\psi(\alpha)$ by $z^{\alpha}=z_{1}^{\alpha_{1}}, \ldots, z_{s}^{\alpha_{s}}, z \in \mathbf{C}^{s}$, in (2.1) gives

$$
\sum_{\alpha \in \mathbf{Z}^{s}} z^{\alpha} t(\alpha \mid X)=\sum_{\beta \in \mathbf{Z}_{+}^{n}} z^{X \beta}=\frac{1}{\prod_{j=1}^{n}\left(1-z^{x^{j}}\right)},
$$

the generating function for $t(\cdot \mid X)$. Similarly, the generating function for $b_{D}(\cdot \mid X)$ is

$$
\sum_{\alpha \in \mathbf{Z}^{s}} z^{\alpha} b_{D}(\alpha \mid X)=\prod_{j=1}^{n}\left(\frac{1-z^{d_{j} x^{j}}}{1-z^{x^{j}}}\right) .
$$

Defining

$$
\mathscr{L}(X)=\left\{X \beta: \beta \in \mathbf{Z}^{n}\right\}, \quad \mathscr{L}_{+}(X)=\left\{X \beta: \beta \in \mathbf{Z}_{+}^{n}\right\}
$$

we clearly have

$$
\operatorname{supp} t(\cdot \mid X)=\left\{\alpha \in \mathbf{Z}^{s}: t(\alpha \mid X) \neq 0\right\}=\mathscr{L}_{+}(X) .
$$

Moreover, as an immediate consequence of our definition we see that when $n=s$ and $\langle X\rangle=\mathbf{R}^{s}$ then

$$
t(\alpha \mid X)= \begin{cases}1, & \alpha \in \mathscr{L}_{+}(X) \\ 0, & \text { otherwise }\end{cases}
$$

In general one has for any $V \subset X$

$$
t(\cdot \mid X)=t(\cdot \mid X \backslash V) * t(\cdot \mid V), \quad b_{D}(\cdot \mid X)=b_{\left.D\right|_{X \backslash V}}(\cdot \mid X \backslash V) * b_{D_{\mid V}}(\cdot \mid V)
$$

where $(g * f)(\alpha)=\sum_{\nu \in \mathbf{Z}^{*}} g(\alpha-\nu) f(\nu)$ denotes discrete convolution of $g$ and $f$ and $\left.D\right|_{V}=\operatorname{diag}\left(d_{i_{1}}, \ldots, d_{i_{m}}\right)$, if $V=\left\{x^{i_{1}}, \ldots, x^{i_{m}}\right\}$. (The continuous analogs of these equations for $B(\cdot \mid X)$ and $T(\cdot \mid X)$ may be found in [6]). In particular, when $V=\left\{x^{i}\right\}(2.10)$ yields

$$
t(\alpha \mid X)=\sum_{j=0}^{\infty} t\left(\alpha-j x^{i} \mid X \backslash\left\{x^{i}\right\}\right)
$$

which combined with (2.9) gives a recursive method for computing $t(\alpha \mid X)$. A similar recursion, of course, holds for $b_{D}(\cdot \mid X)$.

It is easy to see that

$$
\begin{aligned}
& \nabla_{V} t(\alpha \mid X)=t(\alpha \mid X \backslash V), \\
& \nabla_{V} b_{D}(\alpha \mid X)=\nabla_{\left.V D\right|_{V}} b_{\left.D\right|_{X \backslash V}}(\alpha \mid X \backslash V), \quad \alpha \in \mathbf{Z}^{s} .
\end{aligned}
$$

These equations "invert" (2.10) and also have counterparts for the box spline and truncated power.

When $\alpha$ is large (2.11) may be inconvenient for evaluating $t(\alpha \mid X)$. We shall therefore present some other identities for $t(\cdot \mid X)$ which may also be useful. To this end, let $e^{i}=\left(\delta_{i j}\right)_{j=1}^{s}$ denote the $i$ th coordinate vector. Taking the $i$ th partial 
derivative of both sides of (2.6) yields

$$
\begin{aligned}
\sum_{\alpha \in \mathbf{Z}^{s}}\left(\left(\alpha_{i}+1\right) t\left(\alpha+e^{i} \mid X\right)\right) z^{\alpha} & =\sum_{j=1}^{n} x_{i}^{j} z^{x^{j}-e^{i}} \frac{1}{\left(1-z^{x^{1}}\right) \cdots\left(1-z^{x^{j}}\right)^{2} \cdots\left(1-z^{x^{n}}\right)} \\
& =\sum_{j=1}^{n} x_{i}^{j} z^{x^{j}-e^{i}} \sum_{\alpha \in \mathbf{Z}^{s}} z^{\alpha} t\left(\alpha \mid X \cup\left\{x^{j}\right\}\right) \\
& =\sum_{\alpha \in \mathbf{Z}^{s}} z^{\alpha}\left(\sum_{j=1}^{n} x_{i}^{j} t\left(\alpha+e^{i}-x^{j} \mid X \cup\left\{x^{j}\right\}\right)\right) .
\end{aligned}
$$

Hence, in general we get, by setting $\nu \cdot \alpha=\sum_{i=1}^{s} \nu_{i} \alpha_{i}$, the equation

$$
(\nu \cdot \alpha) t(\alpha \mid X)=\sum_{j=1}^{n}\left(X^{T} \nu\right)_{j} t\left(\alpha-x^{j} \mid X \cup\left\{x^{j}\right\}\right)
$$

thereby relating a lower order truncated power to several higher order ones. The usefulness of this formula for certain choices of $X$ will be discussed below.

Our next formula relates discrete truncated powers to the denumerant, i.e., to univariate discrete truncated powers. To this end, choose $\lambda \in \mathbf{Z}^{s}$ such that for every $k \in \mathbf{Z}$

$$
\left|\left\{\alpha \in \mathbf{Z}^{s}: \lambda \cdot \alpha=k\right\} \cap\langle X\rangle_{+}\right|<\infty
$$

where $\langle X\rangle_{+}=\left\{X t: t \in \mathbf{R}_{+}^{n}\right\}$ is the cone spanned by $X$. Then for any function $g: \mathbf{Z} \rightarrow \mathbf{R}$ with finite support one has

$$
\begin{aligned}
\sum_{\alpha \in \mathbf{Z}^{s}} g(\lambda \cdot \alpha) t(\alpha \mid X) & =\sum_{\beta \in \mathbf{Z}_{+}^{n}} g(\lambda \cdot(X \beta))=\sum_{\beta \in \mathbf{Z}_{+}^{n}} g\left(\left(X^{T} \lambda\right) \cdot \beta\right) \\
& =\sum_{k \in \mathbf{Z}} g(k) t\left(k \mid X^{T} \lambda\right) .
\end{aligned}
$$

On the other hand, since

$$
\sum_{\alpha \in \mathbf{Z}^{s}} g(\lambda \cdot \alpha) t(\alpha \mid X)=\sum_{k \in \mathbf{Z}} g(k)\left(\sum_{\lambda \cdot \beta=k} t(\beta \mid X)\right)
$$

we conclude that

$$
t\left(k \mid X^{T} \lambda\right)=\sum_{\lambda \cdot \alpha=k} t(\alpha \mid X), \quad k \in \mathbf{Z} .
$$

Therefore, the discrete Radon transform of a discrete truncated power is a denumerant.

To arrive at another identity for $t(\cdot \mid X)$ we choose $\lambda \in \mathbf{R}^{n}$ and consider the telescoping expansion

$$
\begin{aligned}
1-\lambda^{\beta}= & \left(1-\lambda_{1}^{\beta_{1}}\right)+\left(\lambda_{1}^{\beta_{1}}-\lambda_{1}^{\beta_{1}} \lambda_{2}^{\beta_{2}}\right)+\left(\lambda_{1}^{\beta_{1}} \lambda_{2}^{\beta_{2}}-\lambda_{1}^{\beta_{1}} \lambda_{2}^{\beta_{2}} \lambda_{3}^{\beta_{3}}\right) \\
& +\cdots+\left(\lambda_{1}^{\beta_{1}} \lambda_{2}^{\beta_{2}} \cdots \lambda_{n-1}^{\beta_{n-1}}-\lambda_{1}^{\beta_{1}} \lambda_{2}^{\beta_{2}} \cdots \lambda_{n}^{\beta_{n}}\right) \\
= & \sum_{i=1}^{n} q_{i}(\lambda)\left(1-\lambda_{i}\right)
\end{aligned}
$$


where

$$
q_{r}(\lambda)= \begin{cases}\lambda^{\beta(r-1)} \sum_{l=0}^{\beta_{r}-1} \lambda_{r}^{l}, & \beta_{r}>0 \\ 0, & \beta_{r}=0\end{cases}
$$

and $\beta(r)=\left(\beta_{1}, \ldots, \beta_{r}, 0, \ldots, 0\right)$. Now suppose $X \beta=\nu$ and let $z \in \mathbf{C}^{s}$. We replace each $\lambda_{i}$ by $\lambda_{i} z^{x^{i}}$ in equation (2.15) to obtain the identity

$$
\frac{1-\lambda^{\beta} z^{\nu}}{\prod_{j=1}^{n}\left(1-\lambda_{j} z^{x^{j}}\right)}=\sum_{i=1}^{n} \frac{q_{i}(\lambda, z)}{\prod_{j=1 ; i \neq j}^{n}\left(1-\lambda_{j} z^{x^{j}}\right)}
$$

where

$$
q_{i}(\lambda, z)=\lambda^{\beta(i-1)} z^{\beta_{1} x^{1}+\cdots+\beta_{i-1} x^{i-1}} \sum_{l=0}^{\beta_{i}-1} \lambda_{i}^{l} z^{l x^{i}}
$$

Now, setting $\lambda_{i}=1, i=1, \ldots, n$, we get

$$
\frac{1}{\prod_{j=1}^{n}\left(1-z^{x^{j}}\right)}=\sum_{i=1}^{n} \frac{z^{\beta_{1} x^{1}+\cdots+\beta_{i-1} x^{i-1}}\left(1+z^{x^{i}}+\cdots+z^{\left(\beta_{i}-1\right) x^{i}}\right)}{\prod_{j=1 ; j \neq i}^{n}\left(1-z^{x^{j}}\right)\left(1-z^{\nu}\right)}
$$

Thus we infer from (2.6) after straightforward reindexing and comparison of coefficients that

$$
t(\alpha \mid X)=\sum_{i=1}^{n} \sum_{l=0}^{\beta_{i}-1} t\left(\alpha-X \beta(i-1)-l x^{i} \mid\left(X \backslash\left\{x^{j}\right\}\right) \cup\{X \beta\}\right)
$$

where the interior sum is zero whenever $\beta_{i}=0$. Note that when $\beta=m e^{i},(2.19)$ reduces to

$$
t(\alpha \mid X)=\sum_{l=0}^{m-1} t\left(\alpha-l x^{i} \mid\left(X \backslash\left\{x^{i}\right\}\right) \cup\left\{m x^{i}\right\}\right)
$$

But since for every fixed $\alpha \in \mathbf{Z}$, and $m$ sufficiently large

$$
t\left(\alpha-l x^{i} \mid\left(X \backslash\left\{x^{i}\right\}\right) \cup\left\{m x^{i}\right\}\right)=t\left(\alpha-l x^{i} \mid X \backslash\left\{x^{i}\right\}\right),
$$

(2.20) reduces to $(2.11)$ when $m \rightarrow \infty$, while $(2.20)$ is a trivial identity when $m=1$.

Finally, let us consider the special situation where $X$ consists of repetitions of the elements of some basis $Y=\left\{y^{1}, \ldots, y^{s}\right\} \subset \mathbf{Z}^{s}$ of $\mathbf{R}^{s}$. Specifically, we let $q=\left(q_{1}, \ldots, q_{s}\right)$ and

$$
X=\left\{y^{1}, \ldots, y^{s}\right\}_{q}=\left\{y^{1}, \ldots, y^{1}, \ldots, y^{s}, \ldots, y^{s}\right\}
$$

where each $y^{i}$ is repeated $q_{i}$ times. Clearly, $W=|\operatorname{det} Y| Y^{-1}$ is an integer matrix so that upon substituting $z=\xi^{W}$ below we obtain

$$
\sum_{\alpha \in \mathbf{Z}^{s}} z^{\alpha} t(\alpha \mid X)=\sum_{\alpha \in \mathbf{Z}^{s}} \xi^{W \alpha} t(\alpha \mid X)=\sum_{\alpha \in \mathcal{L}(W)} \xi^{\alpha} t\left(W^{-1} \alpha \mid X\right)
$$


where as before $\mathscr{L}(W)=\left\{W \alpha: \alpha \in \mathbf{Z}^{s}\right\}$. On the other hand, for $n=q_{1}+\cdots+q_{s}$ and $a=|\operatorname{det} Y|$

$$
\begin{aligned}
\sum_{\beta \in \mathbf{Z}_{+}^{n}} z^{X \beta} & =\sum_{\beta \in \mathbf{Z}^{s}} \xi^{W X \beta}=\frac{1}{\left(1-\xi_{1}^{a}\right)^{q_{1}} \cdots\left(1-\xi_{s}^{a}\right)^{q_{s}}} \\
& =\left(\sum_{j \in \mathbf{Z}} \xi_{1}^{j} t\left(j \mid\{a\}_{q_{1}}\right)\right) \cdots\left(\sum_{j \in \mathbf{Z}} \xi_{s}^{j} t\left(j \mid\{a\}_{q_{s}}\right)\right) \\
& =\sum_{\alpha \in \mathbf{Z}^{s}} \xi^{\alpha}\left(t\left(\alpha_{1} \mid\{a\}_{q_{1}}\right) \cdots t\left(\alpha_{s} \mid\{a\}_{q_{s}}\right)\right)
\end{aligned}
$$

where $\{1\}_{l}=\{1, \ldots, 1\}$ is the set consisting of 1 repeated $l$ times. This means that

$$
t(\alpha \mid X)=t\left((W \alpha)_{1} \mid\{a\}_{q_{1}}\right) \cdots t\left(\left(W_{\alpha}\right)_{s} \mid\{a\}_{q_{s}}\right) .
$$

Each of the denumerants $t\left((W \alpha)_{j} \mid\{a\}_{q_{j}}\right)$ can easily be evaluated, see formula (2.28) below.

When $X$ is comprised of more than $s$ distinct directions, the situation is more complicated. Nevertheless, combining formulas (2.19) and (2.23) is sometimes helpful. To illustrate this, consider the example

$$
X=\left\{e^{1}, e^{2}, e^{1}+e^{2}\right\}, \quad e^{i}=\left(\delta_{i j}\right)_{j=1}^{2} .
$$

Using (2.19) for $\beta=(1,1,0)$ we get for $\alpha=\left(\alpha_{1}, \alpha_{2}\right)$

$$
t(\alpha \mid X)=t\left(\alpha \mid e^{2}, e^{1}+e^{2}, e^{1}+e^{2}\right)+t\left(\alpha-e^{1} \mid e^{1}, e^{1}+e^{2}, e^{1}+e^{2}\right)
$$

where both summands are now of type (2.22). Specifically, one obtains from this equation that

$$
\begin{aligned}
t(\alpha \mid X) & =\left(\alpha_{2}-\alpha_{1}\right)_{+}^{0}\left(\alpha_{1}+1\right)_{+}+\left(\alpha_{1}-1-\alpha_{2}\right)_{+}^{0}\left(\alpha_{2}+1\right)_{+} \\
& =\min \left(\alpha_{1}, \alpha_{2}\right)+1
\end{aligned}
$$

where

$$
x_{+}^{k}= \begin{cases}x^{k}, & x \geq 0 \\ 0, & x<0\end{cases}
$$

In this particular case, the discrete truncated power could have been determined directly.

There seems to be no general simple recursive scheme for the evaluation of $t(\alpha \mid X)$ which is independent of $\alpha$. We will therefore employ other means to obtain more information about $t(\cdot \mid X)$. In order to explain what we have in mind, we review some facts about denumerants. First consider the simple example

$$
X_{n}=\{1\}_{n} .
$$

It is easy to verify that

$$
t\left(j \mid X_{n}\right)=\left(\begin{array}{c}
j+n-1 \\
n-1
\end{array}\right), \quad j \geq 0,
$$

(for instance, use (2.11) and induction on $n$ ). Clearly, (2.25) shows that $t\left(\cdot \mid X_{n}\right)$ is a polynomial of degree $n-1$ in $j \in \mathbf{Z}_{+}$, because if we define $P_{n}(t)=(t+n-$ 1) $\cdots(t+1) /(n-1)$ !, one has

$$
t\left(j \mid X_{n}\right)=P_{n}(j), \quad j=0,1,2, \ldots
$$


Moreover, since

$$
P_{n}(j)=\delta_{0 j}, \quad j=0,-1, \ldots,-n+1,
$$

we see that $P_{n}$ and $t\left(j \mid X_{n}\right)$ even agree for $j=-n+1, \ldots, 0,1, \ldots$ In general, denumerants do not exhibit such a simple polynomial structure. In fact, one easily verifies that

$$
t(j \mid k, \ldots, k)=t\left(j \mid k X_{n}\right)= \begin{cases}t\left(l \mid X_{n}\right), & j=l k, l \in \mathbf{Z}, \\ 0, & \text { otherwise }\end{cases}
$$

E. T. Bell [2] clarified the form of denumerants as follows:

THEOREM 2.1 (E. T. BELL). Let a be the least common multiple of $x_{1}, \ldots, x_{n}$ $\in \mathbf{N}$. Then for every fixed $b \in \mathbf{Z}_{+}, 0 \leq b<a, t\left(j a+b \mid x_{1}, \ldots, x_{n}\right)$ is a polynomial of degree $n-1$ as a function of $j \in \mathbf{Z}_{+}$.

This nice result implies that one only has to compute $t\left(j a+b \mid x_{1}, \ldots, x_{n}\right)$, $j=0, \ldots, n-1$, for instance by using (2.11), in order to determine the value of $t\left(j a+b \mid x_{1}, \ldots, x_{n}\right)$ for any $j \in \mathbf{N}$. Bell's result raises the question of characterizing $t(\alpha \mid X)$ for systems of diophantine equations. A particularly interesting special case of this problem arises in the enumeration of magic squares. Following [13], we call an $m \times m$ matrix $A$ with nonnegative integer entries a magic $r$-square if all row and column sums equal $r$. We denote by $H_{m}(r)$ the number of all magic $r$-squares of order $m$. It is natural to ask how $H_{m}(r)$ behaves as a function of $r$ for any given $m$. MacMahon [11] solved this problem for $m \leq 3$. Stanley [13] proved, among other things, that $H_{m}(r)$ is a polynomial of degree $(m-1)^{2}$ in $r \in \mathbf{N}$.

Let us briefly point out how this problem fits into the present context. For this purpose, we define the $2 m \times m^{2}$ matrix with columns

$$
\bar{M}_{m}=\left(\begin{array}{c}
e^{1} \cdots e^{1} e^{2} \cdots e^{2} \cdots e^{m} \cdots e^{m} \\
e^{1} \cdots e^{m} e^{1} \cdots e^{m} \cdots e^{1} \cdots e^{m}
\end{array}\right)
$$

where $e^{i}=\left(\delta_{i j}\right)_{j=1}^{m}$. With any $m \times m$ matrix $A=\left(a_{i j}\right)_{i, j=1}^{m}$, we associate the vector $a=\left(a_{11}, \ldots, a_{1 m}, a_{21}, \ldots, a_{2 m}, \ldots, a_{m 1}, \ldots, a_{m m}\right) \in \mathbf{R}^{m^{2}}$. Therefore, $A$ is a magic $r$-square if and only if $a \in \mathbf{Z}_{+}^{m^{2}}$ and

$$
\bar{M}_{m} a=r \bar{e}
$$

where $\bar{e}=(1, \ldots, 1) \in \mathbf{Z}^{2 m}$. Observe that the $2 m$ equations (2.30) are dependent. These equations are equivalent to

$$
M_{m} a=r e
$$

where now $e=(1, \ldots, 1) \in \mathbf{Z}^{2 m-1}$ and $M_{m}$ is obtained from $\bar{M}_{m}$ by dropping the first row (any row would do just as well). Clearly, $0 \notin\left[M_{m}\right]$ and $M_{m}$ has full rank $2 m-1$ (the $(2 m-1) \times(2 m-1)$ minor consisting of the first $m$ columns of $M_{m}$ and one column from each of the remaining blocks of consecutive $m$ columns is nonsingular). Thus for $s=2 m-1, n=m^{2}$ we have

$$
H_{m}(r)=t\left(r e \mid M_{m}\right) \text {. }
$$

As we shall see, Bell's result as well as some results about $H_{m}(r)$ obtained by Stanley [13] follow from general results for $t(\cdot \mid X)$ which we will now describe. 
3. A characterization of $t(\cdot \mid X)$. The objective of this section is to extend Bell's result mentioned in the previous section to systems of diophantine equations.

Our starting point is the difference relation (2.12) which states that for any $V \subset X$

$$
\nabla_{V} t(\alpha \mid X)=t(\alpha \mid X \backslash V)
$$

Next, we require the following collections of subsets of $X$

$$
\mathscr{Y}(X)=\left\{V \subset X:\langle X \backslash V\rangle \neq \mathbf{R}^{s}\right\}
$$

and also

$$
\mathscr{B}(X)=\left\{Y \subset X:|Y|=s,\langle Y\rangle=\mathbf{R}^{s}\right\} .
$$

Note that in the terminology of matroid theory, $(X, \mathscr{B}(X))$ is a represented matroid and the minimal elements of $\mathscr{Y}(X)$ are its cocircuits (cf. [15]).

In view of (2.8) and (2.9) the relation (3.1) assures that

$$
\nabla_{V} t(\alpha \mid X)=0
$$

for any $\alpha \notin \mathscr{L}_{+}(X \backslash V)$. Also notice that when $V \in \mathscr{Y}(X)$, we have $\operatorname{dim}\langle X \backslash V\rangle \leq$ $s-1$ so that (3.2) actually holds for "most" lattice points in $\mathbf{Z}^{s}$. The relation (3.2) suggests that we consider the following space of lattice functions [6]

$$
\nabla(X)=\left\{f: \mathbf{Z}^{s} \rightarrow \mathbf{C}, \nabla_{V} f=0, V \in \mathscr{Y}(X)\right\} .
$$

To state the main result of this section we find it convenient to use the following terminology. We say that an open cone $\Omega \subset\langle X\rangle_{+}$is an $X$-cone if $\Omega$ is bounded by but not intersected by any of the subspaces $\langle V\rangle, V \subset X, \operatorname{dim}\langle V\rangle=s-1$. We call these subspaces $X$-planes for $T(\cdot \mid X)$. Moreover, we set

$$
Z(X)=\left\{X v: v \in[0,1]^{n}\right\}
$$

and for any set $K \subseteq \mathbf{R}^{s}$

$$
b(K \mid X)=\left\{\alpha \in \mathbf{Z}^{s}:(\alpha+Z(X)) \cap K \neq \varnothing\right\} .
$$

When $K=\{y\}$ we denote this set by $b(y \mid X)$.

Note that in view of (1.4) $Z(X)$ is the support of the box spline $B(\cdot \mid X)$ and $b(\Omega \mid X)$ consists of those lattice points $\alpha$ for which the support of $B(\cdot-\alpha \mid X)$ intersects $\Omega$.

The main result of this section is

THEOREM 3.1. For any $X$-cone $\Omega$, there exists a unique $f_{\Omega}(\cdot \mid X) \in \nabla(X)$ such that

$$
f_{\Omega}(\alpha \mid X)=t(\alpha \mid X), \quad \alpha \in b(\Omega \mid X) .
$$

Moreover, $f_{\Omega}(\cdot \mid X)$ has the following properties: for any $u \in \Omega$ such that

$$
b(u \mid X) \cap \mathscr{L}_{+}(X)=\{0\},
$$

$f_{\Omega}(\cdot \mid X)$ is uniquely determined by

$$
f_{\Omega}(\alpha \mid X)=\delta_{0 \alpha}, \quad \alpha \subset b(u \mid X),
$$


and satisfies the relation

$$
f_{\Omega}(\alpha \mid X)=(-1)^{n-s} f_{\Omega}\left(-\alpha-\sum_{j=1}^{n} x^{j} \mid X\right), \quad \alpha \in \mathbf{Z}^{s} .
$$

The reader should realize that there may be several distinct sets $b(u \mid X)$ which satisfy (3.4).

Observe that properties (3.5) and (3.6) are readily verified for the example (2.24) by using (2.25)--(2.27).

Before proceeding further we make some observations about the condition (3.4) which will prove useful. For this purpose, we introduce the set

$$
\Lambda=\langle X\rangle_{+} \backslash\left(\bigcup_{\alpha \in \mathscr{L}_{+}(X) \backslash\{0\}}\left(\alpha+\langle X\rangle_{+}\right)\right) .
$$

Since for every $\alpha \in \mathscr{L}_{+}(X) \backslash\{0\}$ there is a $y \in X$ such that $\alpha=\alpha_{0}+y$ for some $\alpha_{0} \in \mathscr{L}_{+}(X)$ we may express $\Lambda$ alternatively as

$$
\Lambda=\langle X\rangle_{+} \backslash \bigcup_{y \in X}\left(y+\langle X\rangle_{+}\right)
$$

Recall that from (2.4)

$$
B(x \mid X)=\nabla_{X} T(x \mid X)=T(x \mid X)+\sum_{\substack{V \subset X \\ V \neq \varnothing}} c_{V} T\left(x-\sum_{v \in V} v \mid X\right)
$$

for some integer $c_{V}$. Therefore when $u \in \Lambda,(3.7)$ implies that for $V \neq \varnothing$, $T\left(u-\sum_{v \in V} v \mid X\right)=0$. Hence $(3.8)$ yields

$$
B(u \mid X)=T(u \mid X), \quad u \in \Lambda .
$$

Suppose $u$ belongs to some $X$-cone $\Omega$. Then $u$ satisfies (3.4) if and only if $u$ belongs to $\Lambda$. To see this, observe that (3.10) implies for $u \in \Lambda \cap \Omega$ that $0 \in b(u \mid X)$ because $T(u \mid X)>0$. Moreover, if $\alpha \in b(u \mid X) \cap \mathscr{L}_{+}(X), \alpha \neq 0$ then $u \in \alpha+\langle X\rangle_{+}$ which is excluded by the definition of $\Lambda$. Conversely when $u \in \Omega \backslash \Lambda$ there is an $\alpha \in$ $\mathscr{L}_{+}(X) \backslash\{0\}$ such that $u \in \alpha+\langle X\rangle_{+}$and so $u \in \gamma+Z(X)$, for some $\gamma \in \mathscr{L}_{+}(X) \backslash\{0\}$ which means $\gamma \in b(u \mid X) \cap \mathscr{L}_{+}(X)$.

Furthermore, we also note that since $0 \notin[X]$ there is an $\varepsilon_{0}>0$ such that

$$
\varepsilon_{0} Z(X) \subset \Lambda \text {. }
$$

For our next remark, we introduce the notion of $X$-region of $B(\cdot \mid X)$. This is a region bounded by but not intersected by any of the hyperplanes $\alpha+\langle V\rangle, V \subset X$, $\operatorname{dim} V=s-1, \alpha \in \mathbf{Z}^{s}$. From (3.10) we see that there is a one-to-one correspondence between $X$-cones of $T(\cdot \mid X)$ and $X$-regions of $B(\cdot \mid X)$ whose closure contains the origin. For a given $X$-cone $\Omega$ we will denote the corresponding $X$-region by $\Omega_{0}$.

Finally, we mention a fact often used in our subsequent analysis, namely, $b(u \mid X)$ $=b\left(u^{\prime} \mid X\right)$ if and only if $u$ and $u^{\prime}$ belong to the same $X$-region of $B(\cdot \mid X)$. One should also realize that $\Omega \cap \Lambda$ may contain several different $X$-regions. Therefore when this is the case there are several different sets $b(u \mid X)$ satisfying (3.4). 
In order to formulate a multivariate version of Theorem 2.1 , we require the continuous analog of $\nabla(X)$, namely

$$
D(X)=\left\{f \in \mathscr{D}^{\prime}\left(\mathbf{R}^{s}\right): D_{V} f=0, V \in \mathscr{Y}(X)\right\}
$$

where for any $y \in \mathbf{R}^{s}$,

$$
D_{y} f=\sum_{j=1}^{s} y_{j} \frac{\partial f}{\partial x_{j}}, \quad D_{V} f=\left(\prod_{y \in V} D_{y}\right) f
$$

and $\mathscr{D}^{\prime}\left(\mathbf{R}^{s}\right)$ denotes the space of distributions of all $C^{\infty}\left(\mathbf{R}^{s}\right)$ functions with compact support.

Using standard multi-index notation, let

$$
\Pi_{k}=\left\{\sum_{|\alpha| \leq k} c_{\alpha} x^{\alpha}: c_{\alpha} \in \mathbf{R}\right\}
$$

denote the space of all polynomials of total degree $\leq k$ on $\mathbf{R}^{s}$ and define

$$
d(X)=\max \left\{m: \forall V \subset X,|V|=m \Rightarrow\langle X \backslash V\rangle=\mathbf{R}^{s}\right\} .
$$

We recall the following results from $[\mathbf{5}, \mathbf{7}, \mathbf{8}]$.

THEOREM 3.2 .

$$
\Pi_{d(X)} \subseteq D(X) \subseteq \Pi_{n-s} \text { and } \operatorname{dim} D(X)=|\mathscr{B}(X)| .
$$

We are now prepared to state

THEOREM 3.3. Let $W \subset \mathbf{Z}^{s},|W|=s,\langle W\rangle=\mathbf{R}^{s}$ be chosen so that

$$
\mathscr{L}(W) \subset \mathscr{L}(Y), \quad \forall Y \in \mathscr{B}(X) .
$$

Then for any $\mu \in \mathbf{Z}^{s} \cap\left\{W v: v \in[0,1)^{s}\right\}$ the function $f_{\Omega}(W \cdot+\mu \mid X)$ is a polynomial in $D\left(W^{-1} X\right)$.

As an immediate consequence we state

COROLlARY 3.1. If $X$ is unimodular, i.e.

$$
|\operatorname{det} Y|=1, \quad \forall Y \in \mathscr{B}(X),
$$

then $f_{\Omega}(\cdot \mid X) \in D(X)$.

Note that when $s=1$ the only possible choice of $X$ satisfying (3.14) and (1.1) is given by (2.24). In view of Corollary 3.1 and (2.3) the discrete truncated power and box spline are piecewise polynomials when (3.14) holds.

Somewhat more can be said concerning $t(\alpha \mid X)$ when (3.14) holds.

THEOREM 3.4. Let $X$ satisfy (3.14) and let $\mu \in \mathscr{L}_{+}(X)$ be arbitrary. Then

(i) $t(j \mu \mid X)$ is a polynomial $R_{\mu}(j)$ of exact degree $n-s$ for $j \in \mathbf{Z}_{+}$. Moreover,

(ii) if for some $m \in \mathbf{Z}_{+}, \mu=(1 / m) \sum_{i=1}^{n} x^{i} \in \mathbf{Z}^{s}$ we have $R_{\mu}(-1)=\cdots=$ $R_{\mu}(-m+1)=0$, and

(iii) $R_{\mu}(j)=(-1)^{n-s} R_{\mu}(-j-m), j \in \mathbf{Z}$.

Before proving these results we will apply them to the problem of counting magic squares. 
4. Magic squares. In this section, we apply the above results to the problem of counting the number of magic squares of order $m$ with a fixed row and column sum equal to $r$. We already pointed out in $\S 2$ how this fits into the context of discrete truncated powers. We will adhere to the notation used earlier. Thus, $M_{m}$ is the $s \times n$ matrix where $s=2 m-1, n=m^{2}$ defined in (2.31). The first step in our analysis is

THEOREM 4.1. For any $m \geq 2$

$$
|\operatorname{det} Y|=1, \quad \forall Y \in \mathscr{B}\left(M_{m}\right) \text {. }
$$

Moreover, $d\left(M_{m}\right)=m-1$ and

$$
\left|\mathscr{B}\left(M_{m}\right)\right|=\operatorname{dim} D\left(M_{m}\right)=m^{2 m-2} .
$$

PROOF. The first assertion is a well-known property of the transportation problem in linear programming. It is a consequence of the following lemma due to Heller and Tompkins (cf. [10]).

LEMMA 4.1. Let $A$ be any matrix whose rows can be partitioned into two disjoint sets, $T_{1}$ and $T_{2}$, such that $A, T_{1}$ and $T_{2}$ have the following properties:

(a) Every entry of $A$ is 0,1 or -1 .

(b) Every column contains at most two nonzero entries.

(c) If a column of $A$ contains two nonzero entries having both the same sign then one of the respective rows belongs to $T_{1}$ and the other one belongs to $T_{2}$.

(d) If a column of $A$ contains two nonzero entries having opposite signs then both rows are in $T_{1}$ or both are in $T_{2}$.

Then $A$ is totally unimodular, i.e. all its minors are 0,1 or -1 .

To prove this lemma one observes first that the properties (a), (b), (c), (d) are hereditary, i.e. every submatrix of $A$ has these properties, too. The assertion now follows by induction in the following way. Pick any column of $A$. If it has at most one nonzero entry then the result follows by the induction hypotheses. Otherwise, this column has two nonzero entries. If the respective rows belong to the same group, we add them together producing a zero row and apply induction as before. If the respective rows are in opposite groups $T_{1}$ and $T_{2}$ subtracting one of the rows from the other one, only one nonzero entry is left in this column. It is easy to see that after elimination the matrix still satisfies (a), (b), (c) and (d) so that the induction step proves the lemma.

In view of Theorem 3.2, the assertion (4.2) follows as soon as we prove $\left|\mathscr{B}\left(M_{m}\right)\right|$ $=m^{2 m-2}$. For this purpose, consider the matrix $G_{m}=M_{m} M_{m}^{T}$. Since

$$
\operatorname{det} G_{m}=\sum_{1 \leq i_{1}<\ldots<i_{2 m-1} \leq m^{2}} M_{m}\left(\begin{array}{l}
1, \ldots, 2 m-1 \\
i_{1}, \ldots, i_{2 m-1}
\end{array}\right)^{2}
$$

where for $A=\left(a_{i j}\right)_{i, j=1}^{p, q}$ and any $j_{1}<\cdots<j_{\gamma}, l_{1}<\cdots<l_{\gamma}$

$$
A\left(\begin{array}{c}
j_{1}, \ldots, j_{\gamma} \\
l_{1}, \ldots, l_{\gamma}
\end{array}\right)=\operatorname{det}\left(\begin{array}{ccc}
a_{j_{1} l_{1}} & \cdots & a_{j_{1} l_{\gamma}} \\
\vdots & & \vdots \\
a_{j_{\gamma} l_{1}} & \cdots & a_{j_{\gamma} l_{\gamma}}
\end{array}\right)
$$


we conclude from (4.1) that $\operatorname{det} G_{m}=\left|\mathscr{B}\left(M_{m}\right)\right|$. A direct calculation shows that

$$
G_{m}=\left[\begin{array}{cccccc}
m & \ldots & 0 & 1 & \ldots & 1 \\
\vdots & & \vdots & \vdots & & \vdots \\
0 & \ldots & m & 1 & \ldots & 1 \\
1 & \ldots & 1 & m & \ldots & 0 \\
\vdots & & \vdots & \vdots & & \vdots \\
1 & \ldots & 1 & 0 & \ldots & m
\end{array}\right] .
$$

By elementary row and column eliminations we obtain

$$
\operatorname{det} G_{m}=(-1)^{m}\left(\frac{m-1}{m}\right)^{m} m^{m-1} P\left(\frac{m^{2}}{m-1}\right)
$$

where

$$
P(\lambda)=\operatorname{det}\left[\begin{array}{llr}
1-\lambda & \ldots & 1 \\
\vdots & & \vdots \\
1 & \ldots & 1-\lambda
\end{array}\right]=(-1)^{m} \lambda^{m-1}(\lambda-m)
$$

whence the result follows. The value of $d\left(M_{m}\right)$ is easily obtained. We omit the details.

This leads us to the following result, see Stanley $[\mathbf{1 3}]$.

COROLLARY 4.1. (i) $H_{m}(r)$ is a polynomial in $r$ of exact degree $(m-1)^{2}$,

(ii) $H_{m}(-1)=H_{m}(-2)=\cdots=H_{m}(-m+1)=0$, and

(iii) $H_{m}(-m-j)=(-1)^{m-1} H_{m}(j), j \in \mathbf{Z}$.

PROOF. In view of equation $(2.31), H_{m}(j)=t\left(j e \mid M_{m}\right)$. Therefore Corollary 4.1 follows from Theorem 3.4 and the observation that

$$
\sum_{i=1}^{m^{2}} x^{i}=m e
$$

5. The relationship to multivariate splines. The spaces $D(X), \nabla(X)$ needed for the characterization of $t(\cdot \mid X)$ also play a central role in the discussion of the algebraic properties of $\operatorname{span}\left\{B(\cdot-\alpha \mid X): \alpha \in \mathbf{Z}^{s}\right\}$ (cf. [7, 8]). Let us briefly review the main facts which are relevant for the present context.

Let

$$
A(X)=\left\{z \in \mathbf{C}^{s}: \exists Y \in \mathscr{B}(X), \forall y \in Y, z^{y}=1\right\} .
$$

As pointed out in [7] every $z \in A(X)$ has the form

$$
z=\left(e^{2 \pi i \alpha_{1}^{j} /|\operatorname{det} Y|}, \ldots, e^{2 \pi i \alpha_{s}^{j} /|\operatorname{det} Y|}\right)
$$

for some $Y \in \mathscr{B}(X)$ and some integer $j, 1 \leq j \leq k=|\operatorname{det} Y|$. The vectors $\alpha^{1}, \ldots, \alpha^{k}$ are in $\mathbf{Z}^{s}$ and given by the equations

$$
Y^{T} \alpha^{j}=|\operatorname{det} Y| \mu^{j}, \quad j=1, \ldots, k,
$$

where $\mu^{1}, \ldots, \mu^{k}$ are lattice points in the parallelepiped determined by $Y^{T}$, that is,

$$
\left\{\mu^{1}, \ldots, \mu^{k}\right\}=\left\{Y^{T} v: v \in[0,1)^{s}\right\} \cap \mathbf{Z}^{s} .
$$

We now introduce the set

$$
X_{z}=\left\{y \in X: z^{y}=1\right\}
$$

so that we can state the following theorem. 
THEOREM 5.1. $f \in \nabla(X)$ if and only if

$$
f(\alpha)=\sum_{z \in A(X)} z^{\alpha} p(\alpha \mid z)
$$

where for each $z \in A(X), p(\cdot \mid z)$ is some polynomial in $D\left(X_{z}\right)$ and moreover,

$$
\operatorname{dim} \nabla(X)=\sum_{Y \in \mathscr{B}(X)}|\operatorname{det} Y|
$$

Note that $e=(1, \ldots, 1) \in A(X)$, and $X_{e}=X$, so that in particular

$$
D(X) \subseteq \nabla(X)
$$

and every $f \in \nabla(X)$ has a unique representation $f=P f+E f$ where $P f \in D(X)$ and $E f \in E(X)$, the space of functions $g$ of the form

$$
g(\alpha)=\sum_{z \in A(X) \backslash\{e\}} z^{\alpha} p(\alpha \mid z), \quad p(\cdot \mid z) \in D\left(X_{z}\right) .
$$

Thus, in particular,

$$
D(X)=\nabla(X)
$$

if and only if (3.14) holds.

Next, let

$$
c(X)=\bigcup\left\{\langle V\rangle+\alpha: \alpha \in \mathbf{Z}^{s},|V|=\operatorname{dim}\langle V\rangle=s-1, V \subset X\right\} .
$$

Therefore $c(X)$ is the union of the faces of $\alpha+Z(X), \alpha \in \mathbf{Z}^{s}$. Recall that by our definition the set $b(y \mid X)$ for $y \notin c(X)$ (see $\S 3$ ) identifies those translates of the box spline which are nonzero at the point $y$. The next result from [8] counts the number of lattice points in $b(y \mid X)$.

ThEOREM 5.2. For any $y \notin c(X)$ one has

$$
|b(y \mid X)|=\sum_{Y \in \mathscr{B}(X)}|\operatorname{det} Y|=\operatorname{dim} \nabla(X)=\operatorname{vol}_{s}(Z(X)) .
$$

Moreover, for any sequence $\left\{d_{\alpha}\right\}_{\alpha \in b(y \mid X)}$ there exists a unique $f \in \nabla(X)$ such that

$$
f(\alpha)=d_{\alpha}, \quad \alpha \in b(y \mid X) .
$$

Thus condition (3.5) in Theorem 3.1 uniquely determines the extension $f_{\Omega}(\cdot \mid X)$ of $t(\cdot \mid X)$.

At this point we can derive Theorem 3.3 from Theorem 3.1 (whose proof will appear later).

ProOF OF THEOREM 3.3. We infer from (5.1) that for any $z \in A(X)$ there is a $Y \in \mathscr{B}(X)$ such that

$$
\begin{aligned}
z^{W \beta+\mu} & =z^{\mu} z^{W \beta}=z^{\mu} \exp \left\{2 \pi i\left(\alpha^{k}\right)^{T} W \beta /|\operatorname{det} Y|\right\} \\
& =z^{\mu} \exp \left\{2 \pi i\left(\mu^{k}\right)^{T} Y^{-1} W \beta\right\}=z^{\mu}
\end{aligned}
$$

since by (3.13) $Y^{-1} W \beta \in \mathbf{Z}^{s}$ for all $\beta \in \mathbf{Z}^{s}$. Thus for any $f \in \nabla(X)$ given by (cf. Theorem 5.1, (5.2))

$$
f(\alpha)=\sum_{z \in A(X)} z^{\alpha} p(\alpha \mid z)
$$


we have

$$
g(\beta)=f(W \beta+\mu)=\sum_{z \in A(X)} Q(\beta \mid z)
$$

where $Q(x \mid z)=z^{\mu} p(W x+\mu \mid z)$ and $z^{\mu} p(x+\mu \mid z) \in D\left(X_{z}\right)$ is a polynomial in $x$ of degree $\leq n-s$ (cf. Theorem 3.2). For any $V \in \mathscr{Y}(X)$ it follows that $V \cap X_{z} \in \mathscr{Y}\left(X_{z}\right)$, $z \in A(X)$ and so $D_{V \cap X_{z}} p(\cdot \mid z)=0$. Thus $\left.D_{W-1} Q(\cdot \mid z)\right|_{x}=\left.z^{\mu} D_{V} p(\cdot \mid z)\right|_{W x+\mu}=0$ and we conclude that $g \in D\left(W^{-1} X\right)$. The assertion now follows from Theorem 3.1.

Let us extract from (1.5) more information about the relationship between $t(\cdot \mid X)$ and its continuous counterpart $T(\cdot \mid X)$. To this end, we need the following two facts.

First, we state an analog of Theorem 3.1 for the truncated power function $T(\cdot \mid X)$.

Proposition 5.1. For each $X$-cone, $\Omega$, there exists a unique polynomial $P_{\Omega} \in$ $D(X)$ such that

$$
T(x \mid X)=P_{\Omega}(x), \quad x \in \Omega .
$$

The proof of this result follows from the relation

$$
D_{V} T(\cdot \mid X)=T(\cdot \mid X \backslash V)
$$

(cf. (3.1)), the definition of $T(\cdot \mid X), D(X)$, and Theorem 3.2.

In view of the relation (2.4), Proposition 5.1 also implies that $c(X)$ gives the $X$-planes of the translates $B(\cdot-\alpha \mid X), \alpha \in \mathbf{Z}^{s}$. Thus on any region which is bounded by but not intersected by the hyperplanes in $c(X)$ any function in $\operatorname{span}\left\{B(\cdot-\alpha \mid X): \alpha \in \mathbf{Z}^{s}\right\}$ agrees with some polynomial in $D(X)$. Next we require

PROPOSITION 5.2. (i) The linear operator $F$ defined by

$$
(F f)(x)=\sum_{\alpha \in \mathbf{Z}^{s}} f(\alpha) B(x-\alpha \mid X)
$$

maps $D(X)$ one-to-one and onto itself $[\mathbf{3}, \mathbf{5}]$.

(ii) For any $g \in E(X)$ one has $[8](F g)(x)=0$.

Now recall from [8] that the translates $B(\cdot-\alpha \mid X), \alpha \in \mathbf{Z}^{s}$, are locally linearly independent meaning that $\sum_{\alpha \in \mathbf{Z}^{s}} c_{\alpha} B(x-\alpha \mid X)=0, x \in \Omega$, where $\Omega$ is any domain in $\mathbf{R}^{s}$, implies $c_{\alpha}=0, \alpha \in b(\Omega \mid X)$ if and only if $X$ is unimodular, i.e. (3.14) holds. Thus, in view of (1.5), Propositions 5.1 and 5.2 immediately imply that $t(\cdot \mid X)$ agrees on $b(\Omega \mid X)$ with some polynomial in $D(X)$, if $(3.14)$ is valid. This gives an alternative derivation of Corollary 3.1.

When $X$ is unimodular, that is, under assumption (3.14), additional information about $t(\cdot \mid X)$ can be obtained. To this end, we recall the following construction of a dual basis for the translates of a box spline. For any $v \in \mathbf{R}^{s}$, let $\Phi(x)=B(x+v \mid X)$. Since $\widehat{\Phi}(0)=1$, the expansion

$$
(\widehat{\Phi}(x))^{-1}=\sum_{\alpha \in \mathbf{Z}_{+}^{s}} a_{\alpha}(v) x^{\alpha}
$$

is valid in some neighborhood of zero. Here, as usual, for $f \in L_{1}\left(\mathbf{R}^{s}\right)$ the Fourier transform $\hat{f}$ is defined by

$$
\hat{f}(x)=\int_{\mathbf{R}^{s}} f(y) e^{-i x \cdot y} d y
$$


Setting

$$
\left(L_{v} f\right)(x)=\sum_{|\alpha| \leq n-s} a_{\alpha}(v)(-i)^{|\alpha|} D^{\alpha} f(x)
$$

it was shown in [8] that for $v \notin c(X)$

$$
\left(L_{v} B(\cdot-\beta \mid X)\right)(\alpha+v)=\delta_{\alpha \beta}, \quad \alpha, \beta \subset \mathbf{Z}^{s} .
$$

Choosing $v$ so that $t v \notin c(X), t \in(0,1)$ we may define

$$
(L f)(x)=\sum_{|\alpha| \leq n-s}(-i)^{|\alpha|} a_{\alpha} D_{+}^{\alpha} f(x)
$$

where $D_{+}^{\alpha} f(x)=\lim _{t \rightarrow 0^{+}} D^{\alpha} f(x+t v), a_{\alpha}=a_{\alpha}(0)$, to obtain

$$
(L B(\cdot-\beta \mid X))(\alpha)=\delta_{\alpha \beta}, \quad \alpha, \beta \in \mathbf{Z}^{s} .
$$

Applying $L$ to both sides of relation (1.5) yields

$$
(L T(\cdot \mid X))(\alpha)=t(\alpha \mid X)
$$

Observing that $a_{0}=1$ we obtain

$$
T(\alpha \mid X)+\sum_{0<|\gamma| \leq n-s}(-i)^{|\gamma|} a_{\gamma} D_{+}^{\gamma} T(\alpha \mid X)=t(\alpha \mid X), \quad \alpha \in \mathbf{Z}^{s},
$$

which relates $t(\cdot \mid X)$ directly to the truncated power $T(\cdot \mid X)$, a piecewise polynomial of degree $\leq n-s$.

Now, let $\Omega$ be any $X$-cone and note that for $P_{\Omega}(\cdot)=\left.T(\cdot \mid X)\right|_{\Omega} \in D(X),(5.9)$ yields

$$
P_{\Omega}(x)=\sum_{\alpha \in \mathbf{Z}^{s}}\left(L P_{\Omega}\right)(\alpha) B(x-\alpha \mid X)
$$

We define $f_{\Omega}=L P_{\Omega}$ which by Proposition 5.2 belongs to $D(X)$. Thus (1.5) and the local linear independence of $B(\cdot-\alpha \mid X), \alpha \in \mathbf{Z}^{s}$, provide

$$
f_{\Omega}(\alpha \mid X)=t(\alpha \mid X), \quad \alpha \in b(\Omega \mid X) .
$$

Clearly, for $u \in \Omega, b(u \mid X) \subset b(\Omega \mid X)$ and if $u$ satisfies (3.4) then $t(\alpha \mid X)=\delta_{0 \alpha}, \alpha \in$ $b(u \mid X)$. Thus we have confirmed (3.3)-(3.5) in Theorem 3.1, when $X$ is unimodular.

Moreover, the representation (5.11) allows us to prove Theorem 3.4(i) and (ii) independently of Theorem 3.1 .

PROOF OF THEOREM 3.4. Recalling from $[\mathbf{4}, \mathbf{6}]$ that for any $y \in\langle X\rangle_{+}$

$$
T(t y \mid X)=t_{+}^{n-s} T(y \mid X),
$$

Corollary 3.1 and (5.11) show that for any $\mu \in \mathscr{L}_{+}(X), t(j \mu \mid X)$ is a polynomial in $j \in \mathbf{Z}_{+}$of exact degree $n-s$ which proves (i). If $\mu=(1 / m) \sum_{i=1}^{n} x^{i} \in \mathbf{Z}^{s}$ and $\Omega$ is any $X$-cone we can find $\varepsilon_{0}>0$ such that $u_{\Omega}=X v, v \in\left[0, \varepsilon_{0}\right]^{s}$ satisfies (3.4), see (3.11). Hence

$$
(m-j) \mu+u_{\Omega}=\frac{m-j}{m} \sum_{i=1}^{n} x^{i}+X v=X w,
$$

where $w \in[0,1]^{n}$, whenever $j=1, \ldots, m-1$, i.e.

$$
-j \mu \in b\left(u_{\Omega} \mid X\right), \quad j=1, \ldots, m-1 .
$$


This proves (ii); (iii) follows from (3.6) of Theorem 3.1.

Even when $X$ is not unimodular equation (1.5) and Theorem 3.1 still yield useful information about $t(\cdot \mid X)$. We conclude, as above, for $x \in \Omega$, a fundamental $X$-cone, that

$$
\begin{aligned}
P_{\Omega}(x) & =T(x \mid X)=\sum_{\alpha \in b(\Omega \mid X)} t(\alpha \mid X) B(x-\alpha \mid X) \\
& =\sum_{\alpha \in b(\Omega \mid X)} f_{\Omega}(\alpha \mid X) B(x-\alpha \mid X) \\
& =\sum_{\alpha \in \mathbf{Z}^{s}} P f_{\Omega}(\alpha \mid X) B(x-\alpha \mid X), \quad x \in \Omega,
\end{aligned}
$$

where as before for $f \in \nabla(X)$ its polynomial part in $D(X)$ is denoted by $P f$. Taking into account that

$$
(q(D) \widehat{B}(\cdot \mid X))(2 \pi \alpha)=0, \quad \alpha \in \mathbf{Z}^{s} \backslash\{0\}, q \in D(X),
$$

see [5], Poisson's summation formula converts (5.14) into

$$
P_{\Omega}(x)=\left(P f_{\Omega}(-i D+x \mid X) \widehat{B}(\cdot \mid X)\right)(0) .
$$

Expanding $P f_{\Omega}$ in a Taylor series around $x$ and noting that

$$
\widehat{B}(0 \mid X)=1
$$

the relation (5.16) yields for $x \in \Omega$

$$
T(x \mid X)=P f_{\Omega}(x \mid X)+\sum_{0<|\nu| \leq n-s} D^{\nu} P f_{\Omega}(x \mid X)(-i)^{|\nu|} D^{\nu} \widehat{B}(0 \mid X) / \nu !
$$

Thus we have

PROPOSITION 5.3. The leading homogeneous terms of $P f_{\Omega}(\cdot \mid X)$ agree on $\Omega$ with $T(\cdot \mid X)$.

As a final remark, before proving Theorem 3.1, we recall from [7] that for any $y \notin c(X)$ there exist coefficients $\left\{c_{\beta}\right\}, \beta \in b(y \mid X)$ such that for

$$
\Phi(x)=\sum_{\beta \in b(y \mid X)} c_{\beta} B(x+\beta \mid X),
$$

the operator

$$
(S f)(x)=\sum_{\alpha \in \mathbf{Z}^{s}} f(\alpha) \Phi(x-\alpha)
$$

satisfies

$$
S Q=Q, \quad Q \in D(X) .
$$

Hence, for any $x \in \Omega$

$$
\begin{aligned}
T(x \mid X) & =P_{\Omega}(x)=\left(S P_{\Omega}\right)(x)=\sum_{\alpha \in \mathbf{Z}^{s}} P_{\Omega}(\alpha \mid X) \Phi(x-\alpha) \\
& =\sum_{\alpha \in \mathbf{Z}^{s}}\left(\sum_{\beta \in b(y \mid X)} c_{\beta} P_{\Omega}(\alpha+\beta \mid X)\right) B(x-\alpha \mid X) .
\end{aligned}
$$


Thus, we infer from (5.14) the representation

$$
P f_{\Omega}(\alpha \mid X)=\sum_{\beta \in b(y \mid X)} c_{\beta} P_{\Omega}(\alpha+\beta \mid X), \quad \alpha \in \mathbf{Z}^{s} .
$$

6. Proof of Theorem 3.1. The proof of Theorem 3.1 relies on Theorem 5.2 and various properties of the sets $b(y \mid x), y \notin c(X)$, which will be developed along with the proof. We proceed by induction on $n$. For $n=s$, let

$$
f_{\Omega}(\alpha \mid X)= \begin{cases}1, & \alpha \in \mathscr{L}(X), \\ 0, & \text { otherwise. }\end{cases}
$$

Clearly, $\nabla_{y} f_{\Omega}(\alpha \mid X)=0, \alpha \in \mathbf{Z}^{s}, y \in X$, so that $f_{\Omega}(\cdot \mid X) \in \nabla(X)$. Moreover, by (2.9) $t(\alpha \mid X)=f_{\Omega}(\alpha \mid X), \alpha \in \mathscr{L}_{+}(X)$. The assertion follows for $n=s$ as soon as we show that

$$
b(\Omega \mid X) \cap \mathscr{L}(X)=\mathscr{L}_{+}(X) .
$$

To see this it is sufficient to note that $\Omega=\operatorname{int}\langle X\rangle_{+}$is partitioned by the sets $Z(X)+X \beta=\left\{X(v+\beta): v \in[0,1]^{s}\right\}, \beta \in \mathbf{Z}_{+}^{s}$.

Now let $n>s$. By Theorem 5.2 there exists a unique function $g_{u}(\cdot \mid X) \in \nabla(X)$ satisfying

$$
g_{u}(\alpha \mid X)=\delta_{0 \alpha}, \quad \alpha \in b(u \mid X)
$$

Recall from the remarks following Theorem 3.1 that for every $X$-cone $\Omega$, there is a subset of $\Omega$ which we call $\Omega_{0}$ with the property that every element of $\Omega_{0}$ is on the same side as the origin of every $X$-plane. Thus $\Omega_{0}$ is at the tip of $\Omega$. We will now show that for any $u$ satisfying $(3.4) g_{u}(\cdot \mid X)$ agrees with $t(\cdot \mid X)$ on $b(\Omega \mid X)$. For this purpose, it is sufficient to assume that $u \in \Omega_{0}$ since then Theorem 5.2 would insure that for any $u$ satisfying $(3.4) g_{u}(\cdot \mid X)$ equals $t(\cdot \mid X)$ on $b(\Omega \mid X)$.

LEMMA 6.1. Let $y \in X$ such that $\langle X \backslash\{y\}\rangle=\mathbf{R}^{s}$, $u$ in some $X$-cone $\Omega$, and $b(u \mid X) \cap \mathscr{L}_{+}(X)=\{0\}$.

(i) If $0 \in b(u \mid X \backslash\{y\})$ then

$$
\nabla_{y} g_{u}(\cdot \mid X)=g_{u}(\cdot \mid X \backslash\{y\}) .
$$

(ii) If $0 \notin b(u \mid X \backslash\{y\})$, then

$$
\nabla_{y} g_{u}(\cdot \mid X)=0
$$

ProOF. Clearly, $\nabla_{y} g_{u}(\cdot \mid X) \in \nabla(X \backslash\{y\})$. The proof of (i) and (ii) are now immediate consequences of Theorem 5.2 and the inclusion

$$
b(u \mid X \backslash\{y\}) \cup(b(u \mid X \backslash\{y\})-y) \subseteq b(u \mid X)
$$

which follows from the definition of $b(u \mid X)$.

To make use of this lemma, we combine (3.4) with (6.1) and obtain

$$
t(\alpha \mid X)-g_{u}(\alpha \mid X)=0, \quad \alpha \in b(u \mid X) .
$$

We have to show that (6.4) and our induction hypothesis imply that

$$
g_{u}(\alpha \mid X)=t(\alpha \mid X), \quad \alpha \in b(\Omega \mid X),
$$

which would prove (3.3) and (3.5) for $f_{\Omega}(\cdot \mid X)=g_{u}(\cdot \mid X)$. In order to verify (6.5) we will extend the validity of (6.4) step by step to "neighboring" sets $b\left(u^{\prime} \mid X\right), u^{\prime} \in \Omega$. 
To this end, we recall that the restriction of any linear combination

$$
\sum_{\alpha \in \mathbf{Z}^{s}} c_{\alpha} B(x-\alpha \mid X)
$$

to any $X$-region is a polynomial in $D(X)$. Any two $X$-regions $\Gamma_{1}, \Gamma_{2}$ are called adjacent if $\bar{\Gamma}_{1} \cap \bar{\Gamma}_{2} \subset c(X)$ and $\operatorname{vol}_{s-1}\left(\bar{\Gamma}_{1} \cap \bar{\Gamma}_{2}\right)>0$. We say that a vector $y \in \mathbf{R}^{s}$ is parallel to some hyperplane $H \subset \mathbf{R}^{s}$ whenever $H=H+y$. Moreover, a region $\Gamma$ is said to lie between two parallel hyperplanes $H_{i}=\left\{x: a \cdot x=c_{i}\right\}, i=1,2$, if $\left(a \cdot x-c_{1}\right)\left(a \cdot x-c_{2}\right)<0$, for all $x \in \Gamma$.

LeMmA 6.2. Suppose $\Gamma_{1}, \Gamma_{2}$ are two adjacent $X$-regions separated by the hyperplane $H \subset c(X)$. Let $z^{i} \in \Gamma_{i}, i=1,2$. Then for any $\alpha \in b\left(z^{2} \mid X\right) \backslash b\left(z^{1} \mid X\right)$ and any $y \in X$ not parallel to $H$ there exists $\sigma \in\{ \pm 1\}$ such that $\alpha+\sigma y \in b\left(z^{1} \mid X\right)$. The sign of $\sigma$ is determined by the condition that $\Gamma_{2}$ is between $H-\sigma y$ and $H$. Moreover, if $B(\cdot-\alpha \mid X)$ is continuous on $\bar{\Gamma}_{1} \cup \bar{\Gamma}_{2}$ then one has

$$
\sigma= \begin{cases}1, & \text { if } \alpha \in b\left(z^{2} \mid X \backslash\{y\}\right)-y, \\ -1, & \text { if } \alpha \in b\left(z^{2} \mid X \backslash\{y\}\right) .\end{cases}
$$

ProOF. By assumption $H \cap \bar{\Gamma}_{1}=H \cap \bar{\Gamma}_{2}$ is an $s-1$ face of $\alpha+Z(X)$. Thus it can be represented as $\alpha+p+Z\left(Y^{\prime}\right)$ for some $Y^{\prime}=\left\{y^{1}, \ldots, y^{s-1}\right\} \subset X$ and $p=\sum_{x \notin Y^{\prime}} \delta_{x} x$, where $\delta_{x}=0$ or 1 . Suppose $y \in X$ is not parallel to $Y^{\prime}$, that is, $Y=\{y\} \cup Y^{\prime} \in \mathscr{B}(X) . \bar{\Gamma}_{2}$ is intersected by either the parallelepiped generated by $H \cap \bar{\Gamma}_{2}$ and $y$ or by $H \cap \bar{\Gamma}_{2}$ and $-y$. These sets are given respectively by $\alpha+p+Z(Y)$ and $\alpha+p-y+Z(Y)$. Since $\Gamma_{2}$ is an $X$-region any parallelepiped intersecting it must also contain it. Thus $z^{2} \in \beta+Z(Y)$ for some $\beta \in \mathbf{Z}^{s}$. Define

$$
\sigma= \begin{cases}1, & \beta \notin H, \\ -1, & \beta \in H .\end{cases}
$$

Observe that $\alpha+p \in H$ while $\alpha+p-y \notin H$ since $y$ is not parallel to $H$. Hence the parallelepipeds mentioned above are $\beta+Z(Y)$ and $\beta+\sigma y+Z(Y)$ which means $z^{1}$ must be contained in $\beta+\sigma y+Z(Y)$. Next, observe that if $\beta \notin H$, that is, $\Gamma_{1} \subset \alpha+p+Z(Y)$, then $\Gamma_{1}$ must be between the hyperplanes $H$ and $H+y$. However, if $\delta_{y}=0$ we see that $H$ and $H+y$ are in $\alpha+Z(X)$. Since by assumption $\Gamma_{1}$ is not in $\alpha+Z(X)$ we conclude $\delta_{y}=0$ implies $\beta \in H$. Similarly, when $\delta_{y}=1$ we get $H$ and $H-y$ in $\alpha+Z(X)$ while if $\beta \in H$ then $\Gamma_{1}$ is between $H$ and $H-y$. Thus $\delta_{y}=0$ if and only if $\beta \in H$.

We can now show that $z^{1} \in \alpha+\sigma y+Z(X)$, thereby establishing the first assertion of the lemma. When $\beta \in H$, that is, $\sigma=-1$, then

$$
\beta+\sigma y+Z(Y)=\alpha+p-y+Z(Y)
$$

which is contained in $\alpha-y+Z(X)$. In the other case, $\beta \notin H$, we have $\delta_{y}=1$ so that $p-y \in Z\left(X \backslash Y^{\prime}\right)$ and

$$
\beta+\sigma y+Z(Y)=\alpha+p-y+y+Z(Y)
$$

is now contained in $\alpha+y+Z(X)$. Thus in all cases $\alpha+\sigma y \in b\left(z^{1} \mid X\right)$.

For the second part of the assertion we note that the continuity assumption on $B(\cdot \mid X)$ implies that $D_{y} B(\cdot-\alpha \mid X)$ does not vanish identically on $\Gamma_{2}$. Since

$$
D_{y} B(\cdot \mid X)=B(\cdot \mid X \backslash\{y\})-B(\cdot-y \mid X \backslash\{y\})
$$


we conclude that

$$
\alpha \in b\left(z^{2} \mid X \backslash\{y\}\right) \cup\left(b\left(z^{2} \mid X \backslash\{y\}\right)-y\right)
$$

because $b(z \mid X \backslash\{y\})$ does not change for $z \in \Gamma_{2}$. Now $\alpha \in b\left(z^{2} \mid X \backslash\{y\}\right)$ means that $\Gamma_{2} \subset \alpha+Z(X \backslash\{y\})$. Since every face of $\alpha+Z(X \backslash\{y\})$ has the form $\alpha+\sum_{x \notin V} \delta_{x} x+$ $Z(V), V \subset X \backslash\{y\}, \operatorname{dim}\langle V\rangle=s-1$ we conclude that in the representation for $H \cap \bar{\Gamma}_{2}$, $\delta_{y}=0$ so that $\beta \in H$. Similarly $\alpha \in b\left(z^{2} \mid X \backslash\{y\}\right)-y$ implies $\delta_{y}=1$ and $\beta \notin H$ which by (6.6) completes the proof.

We are now ready to prove (3.3) and (3.5) with $f_{\Omega}=g_{u}$. We assume inductively that Theorem 3.5 holds for all $n<|X|$ and show that for any $y \in X$

$$
\nabla_{y}\left(g_{u}(\alpha \mid X)-t(\alpha \mid X)\right)=0, \quad \alpha \in b(\Omega \mid X \backslash\{y\}) .
$$

We prove this equation by distinguishing two cases: when $0 \in b(u \mid X \backslash\{y\})$ then (6.7) follows from Lemma 6.1, (2.12) and our induction assumption because whenever $\Omega$ is an $X$-cone it is surely contained in an $X \backslash\{y\}$-cone. In the case that $0 \notin b(u \mid X \backslash\{y\})$ we will show that

$$
b(\Omega \mid X \backslash\{y\}) \cap \mathscr{L}_{+}(X \backslash\{y\})=\varnothing .
$$

To accomplish this, let us point out first that $\Omega \cap\langle X \backslash\{y\}\rangle_{+}=\varnothing$. Suppose to the contrary that there is $x \in \Omega \cap\langle X \backslash\{y\}\rangle_{+}$. We can then find $m \in \mathbf{N}$ such that $x / m \in \Omega_{0}$ (see the discussion after Theorem 3.1). Since for any $u \in \Omega_{0}$, $b(u \mid X \backslash\{y\})=b(x / m \mid X \backslash\{y\})$ we conclude $0 \notin b(x / m \mid X \backslash\{y\})$. However, $b(u \mid X)=$ $b(x / m \mid X), u \in \Omega_{0}$, and $0 \in b(u \mid X)$ so that $x / m=(X \backslash\{y\}) t^{1}+t y$ for some $t^{1} \in[0,1]^{s-1}$ and $t \in[0,1], t>0$, otherwise we would have $0 \in b(x / m \mid X \backslash\{y\})$. This gives $x / m \notin\langle X \backslash\{y\}\rangle_{+}$which is a contradiction.

Now, to prove (6.8) we again suppose to the contrary that there is an $\alpha \in$ $b(\Omega \mid X \backslash\{y\}) \cap \mathscr{L}_{+}(X \backslash\{y\})$. Thus there exists $w \in \Omega$ such that for some $t^{1} \in$ $[0,1]^{n-1}, w=\alpha+(X \backslash\{y\}) t^{1} \in \Omega$. Since $\alpha \in \mathscr{L}_{+}(X \backslash\{y\})$ this implies $w \in$ $\langle X \backslash\{y\}\rangle_{+}$contradicting the fact that $\Omega \cap\langle X \backslash\{y\}\rangle_{+}=\varnothing$ which proves (6.8). Finally, combining (2.8), (6.8) and Lemma 6.1(ii) provides (6.7) in this case too.

Now let $\Gamma_{1}, \Gamma_{2}$ be any two adjacent $X$-regions in $\Omega$ and let $z^{i} \in \Gamma_{i}, i=1,2$. In order to verify $(6.5)$ it is sufficient to prove the following

Claim. Suppose

$$
t(\alpha \mid X)=g_{u}(\alpha \mid X), \quad \alpha \in b\left(z^{1} \mid X\right)
$$

Then

$$
t(\alpha \mid X)=g_{u}(\alpha \mid X), \quad \alpha \in b\left(z^{2} \mid X\right) .
$$

In fact, starting with $z^{1}=u$ this would, in view of (6.4), allow us to extend the validity of (6.4) step by step exhausting $b(\Omega \mid X)$.

To prove the above claim let $H \subset c(X)$ denote the $(s-1)$-hyperplane separating $\Gamma_{1}$ and $\Gamma_{2}$ and suppose $\alpha \in b\left(z^{2} \mid X\right) \backslash b\left(z^{1} \mid X\right)$. We have to distinguish two cases. First suppose $B(\cdot-\alpha \mid X)$ is discontinuous across $H$. Then there exists $y \in X$ such that $\langle X \backslash\{y\}\rangle \neq \mathbf{R}^{s}$, and $y$ is not parallel to $H$. Since $\langle X\rangle=\mathbf{R}^{s}, y$ is not parallel to $\langle X \backslash\{y\}\rangle$ which in turn is parallel to $H$. Furthermore, $\langle X \backslash\{y\}\rangle$ is not a separating hyperplane for any $\Gamma^{1}, \Gamma^{2}$ since it forms a boundary of $\langle X\rangle_{+}$so that $H \neq\langle X \backslash\{y\}\rangle$. Now suppose $\alpha$ belongs to $H$. By choice $\Gamma_{2}$ is in $\alpha+Z(X)$ so that $\Gamma_{2}$ lies between $H$ and $H+y$. Thus Lemma 6.2 gives $\alpha-y \in b\left(z^{1} \mid X\right)$. Since $\alpha \in H$ and therefore $\alpha \notin\langle X \backslash\{y\}\rangle$ we get by (3.1) and (2.8) $\nabla_{y} t(\alpha \mid X)=t(\alpha \mid X \backslash\{y\})=0$ while 
$\nabla_{y} g_{u}(\alpha \mid X)=0$ because $\{y\} \in \mathscr{Y}(X)$. Since by assumption (6.9) $t(\alpha-y \mid X)=$ $g_{u}(\alpha-y \mid X)$ this implies $t(\alpha \mid X)=g_{u}(\alpha \mid X) . \Gamma_{2}$ lies between $H$ and $H+y$ or $H$ and $H-y$. Thus when $\alpha \notin H$ it must be between $H$ and $H-y$. Consequently, Lemma 6.2 gives $\alpha+y \in b\left(u^{1} \mid X\right) . Z(X)$ has parallel faces contained in $\alpha+\langle X \backslash\{y\}\rangle_{+}$and $\alpha+y+\langle X \backslash\{y\}\rangle_{+}$and $H$ contains one of them. Hence in this case $\alpha+y \in H$. As before we conclude $\nabla_{y} t(\alpha+y \mid X)=\nabla_{y} g_{u}(\alpha+y \mid X)=0$ and again since by assumption (6.9) $t(\alpha+y \mid X)=g_{u}(\alpha+y \mid X)$ also $t(\alpha \mid X)=g_{u}(\alpha \mid X)$, proving the claim under the assumption that $B(\cdot-\alpha \mid X)$ is discontinuous across $H$.

If, on the other hand, $B(\cdot-\alpha \mid X)$ is continuous on $\Gamma_{1} \cup \Gamma_{2}$ the reasoning in the proof of Lemma 6.2 assures that

$$
\alpha \in b\left(z^{2} \mid X \backslash\{y\}\right) \cup\left(b\left(z^{2} \mid X \backslash\{y\}\right)-y\right)
$$

where $y \in X$ is any direction not parallel to $H$. If $\alpha \in b\left(z^{2} \mid X \backslash\{y\}\right)$ Lemma 6.2 says that $\alpha-y \in b\left(z^{1} \mid X\right)$ so that $g_{u}(\alpha \mid X)=t(\alpha \mid X)$ follows from the assumption (6.9) and (6.7). If $\alpha \in b\left(z^{2} \mid X \backslash\{y\}\right)-y$, that is, $\alpha+y \in b\left(z^{2} \mid X \backslash\{y\}\right)$, Lemma 6.2 implies $y+\alpha \in b\left(z^{1} \mid X\right)$. Again (6.9) combined with (6.7) yields $g_{u}(\alpha \mid X)=t(\alpha \mid X)$. This proves the claim and therefore (3.3), (3.5) in Theorem 3.1.

It remains to verify the reciprocity relation (3.6). Again we will proceed by induction on $n$. Note that for $n=s,\langle X\rangle=\mathbf{R}^{s}, \alpha \in \mathscr{L}(X)$ if and only if $-\alpha-$ $\sum_{j=1}^{s} x^{j} \in \mathscr{L}(X)$ so that (3.6) trivially holds for

$$
f_{\Omega}(\alpha \mid X)= \begin{cases}1, & \alpha \in \mathscr{L}(X), \\ 0, & \alpha \notin \mathscr{L}(X) .\end{cases}
$$

Now suppose (3.6) holds for any strict subset $X^{\prime}$ of $X$ where $|X|=n>s$ and let

$$
Q(\alpha)=g_{u}(\alpha \mid X)-(-1)^{n-s} g_{u}\left(-\alpha-\sum_{j=1}^{n} x^{j} \mid X\right)
$$

where $g_{u}(\cdot \mid X) \in \nabla(X)$ is defined by (6.1) and hence coincides with $f_{\Omega}(\cdot \mid X)$ by (3.5) of Theorem 3.1. If for some $y=x^{l} \in X,\langle X \backslash\{y\}\rangle \neq \mathbf{R}^{s}$, then $\nabla_{y} Q(\alpha)=0, \alpha \in \mathbf{Z}^{s}$, because $\{y\} \in \mathscr{Y}(X)$. On the other hand, if $\langle X \backslash\{y\}\rangle=\mathbf{R}^{s}$ and $0 \in b(u \mid X \backslash\{y\})$ Lemma 6.1(i) gives

$$
\begin{aligned}
\nabla_{y} Q(\alpha)=g_{u}(\alpha \mid X \backslash\{y\})-(-1)^{n-s}\left\{g_{u}\left(-\alpha-\sum_{i=1}^{n} x^{i} \mid X\right)\right. \\
\left.-g_{u}\left(-\alpha-\sum_{i=1 ; i \neq l}^{n} x^{i} \mid X\right)\right\} \\
=g_{u}(\alpha \mid X \backslash\{y\})-(-1)^{n-s-1} g_{u}\left(-\alpha-\sum_{i=1 ; i \neq l}^{n} x^{i} \mid X \backslash\{y\}\right) \\
=0
\end{aligned}
$$

where we have used our induction hypothesis in the last step. Furthermore, when $\langle X \backslash\{y\}\rangle=\mathbf{R}^{s}$ and $0 \notin b(u \mid X \backslash\{y\})$ Lemma 6.1(ii) assures that $\nabla_{y} Q(\alpha)=0$, $\alpha \in \mathbf{Z}^{s}$. Thus under all circumstances

$$
\nabla_{y} Q=0, \quad y \in X
$$


Therefore $Q$ is constant on every lattice $\mathscr{L}(V), V \in \mathscr{B}(X)$, that is,

$$
Q \in \nabla(V), \text { for all } V \in \mathscr{B}(X) \text {. }
$$

Since by Theorem 3.3 of $[8], Z(X)$ can be decomposed into translates of the parellelepipeds $Z(V)=\left\{V t: t \in[0,1]^{s}\right\}, V \in \mathscr{B}(X)$, there is some $Y \in \mathscr{B}(X)$ such that

$$
0 \in b(u \mid Y) \text {. }
$$

We need to consider the lattice points in the half open parallelepiped generated by $Y$. For this purpose we let $\left\{\mu^{1}, \ldots, \mu^{l}\right\}=\mathbf{Z}^{s} \cap\left\{Y t: t \in[0,1)^{s}\right\}$. Recall from $[\mathbf{7}]$ that the sets

$$
Z_{j}=\mu^{j}+\mathscr{L}(Y), \quad j=1, \ldots, l,
$$

form a partition of $\mathbf{Z}^{s}$ and that $l=|\operatorname{det} Y|$. We will show next that for each $j=1, \ldots, l$ there is an $\alpha^{j} \in Z_{j}$ such that $Q\left(\alpha^{j}\right)=0$. Thus we would conclude from (6.12) and Theorem 5.2 that $Q=0$ which advances the induction hypothesis. We base this claim about $Q$ on

LEMma 6.3. Suppose $Y \in \mathscr{B}(X)$ satisfies (6.13). Let $W(X)=\{z \in X: 0 \notin$ $b(u \mid X \backslash\{z\})\}$. Then for each $j=1, \ldots, l$ there exists $\alpha \in Z_{j}$ such that

$$
\left\{\alpha,-\alpha-\sum_{j=1}^{n} x^{j}+\sum_{w \in W(X)} \sigma_{z} w\right\} \subset b(u \mid X) \backslash\{0\}
$$

holds for some $\sigma_{z} \in\{-1,1\}, z \in W(X)$.

Before proving this result, let us observe that $Q(\alpha)=0$ for any $\alpha \in Z_{j}$ satisfying (6.14). In fact, we have for $\alpha \in b(u \mid X) \backslash\{0\}$ that $g_{u}(\alpha \mid X)=0$ by the definition (6.1). Hence

$$
\begin{aligned}
Q(\alpha) & =g_{u}(\alpha \mid X)-(-1)^{n-s} g_{u}\left(-\alpha-\sum_{j=1}^{n} x^{j} \mid X\right) \\
& =-(-1)^{n-s} g_{u}\left(-\alpha-\sum_{j=1}^{n} x^{j} \mid X\right) .
\end{aligned}
$$

From Lemma 6.1(ii) and the definition of $W(X)$ we infer that

$$
Q(\alpha)=-(-1)^{n-s} g_{u}\left(-\alpha-\sum_{j=1}^{n} x^{j}+\sum_{z \in W(X)} \sigma_{z} z \mid X\right)
$$

which by (6.14) in Lemma 6.3 gives $Q(\alpha)=0$, again by the definition of $g_{u}(\cdot \mid X)$. Now (6.11) implies $Q$ vanishes everywhere on $Z_{j}$. Since $j$ was arbitrary we conclude that $Q$ vanishes identically which was to be shown.

Thus it remains to prove Lemma 6.3. To this end, note first that $X t=x$ holds for some $t \in[0,1]^{n}$ if and only if $X(1-t)=\sum_{j=1}^{n} x^{j}-x$, i.e. (cf. (1.4))

$$
B(x \mid X)=B\left(\sum_{j=1}^{n} x^{j}-x \mid X\right) .
$$


Hence we observe that $B(u-\alpha \mid X) \neq 0$ if and only if $B\left(\sum_{j=1}^{n} x^{j}-u+\alpha \mid X\right) \neq 0$ proving that

$$
b(-u \mid X)=-b(u \mid X)-\sum_{j=1}^{n} x^{j} .
$$

Next we will prove Lemma 6.3 first under the assumption

$$
W(X)=\varnothing .
$$

In view of (6.15) this is equivalent to showing that for each $j=1, \ldots,|\operatorname{det} Y|$

$$
Z_{j} \cap(b(u \mid X) \cap b(-u \mid X)) \neq \varnothing
$$

because $0 \notin b(-u \mid X)$. Since $Z(X)$ is convex this is equivalent to saying that

$$
Z_{j} \cap(-\operatorname{int} Z(X)) \neq \varnothing \text {. }
$$

To prove (6.18) we recall from [3] that the condition $W(X)=\varnothing$ implies $B(\cdot \mid X)$ is continuous and that

$$
|\operatorname{det} Y|^{-\dot{1}}=\sum_{\alpha \in \mathscr{L}(Y)} B(x-\alpha \mid X), \quad x \in \mathbf{R}^{s} .
$$

Thus, in particular

$$
1=|\operatorname{det} Y| \sum_{\alpha \in \mathscr{L}(Y)} B\left(-\mu^{j}-\alpha \mid X\right)=|\operatorname{det} Y| \sum_{\alpha \in Z_{j}} B(-\alpha \mid X) .
$$

From this and the continuity of $B(\cdot \mid X)$ equation (6.18) is immediate. Hence we have proved Lemma 6.3 under the assumption that $W(X)=\varnothing$.

For the general case we introduce the set $\widehat{X}=X \cup W(X)$. Clearly this set satisfies (6.16), i.e. $W(\widehat{X})=\varnothing$. Hence for any $j, 1 \leq j \leq l$, there exists $\alpha^{\prime} \in Z_{j}$ and $t^{1}, t^{2} \in[0,1]^{n}$ such that

$$
\alpha^{\prime}+\widehat{X} t^{1}=u, \quad \alpha^{\prime}+\widehat{X} t^{2}=-u .
$$

Since $0 \notin b(-u \mid X)$ (because $0 \notin[\widehat{X}]$ ) we have $\alpha^{\prime} \neq 0$. We may write the first relation in $(6.20)$ as

$$
\alpha^{\prime}+(X \backslash W(X)) \tau+\sum_{w \in W(X)}\left(\tau_{w}^{\prime}+\tau_{w}^{\prime \prime}\right) w=u
$$

for $\tau \in[0,1]^{n-|W(X)|}, \tau_{w}^{\prime}, \tau_{w}^{\prime \prime} \in[0,1]$. Now let $W(X)=V_{1} \cup V_{2}$, where $V_{1}=\{w \in$ $\left.W(X): \tau_{w}^{\prime}+\tau_{w}^{\prime \prime} \leq 1\right\}, V_{2}=\left\{w \in W(X): \tau_{w}^{\prime}+\tau_{w}^{\prime \prime}>1\right\}$ so that

$$
u=\alpha^{\prime}+(X \backslash W(X)) \tau+\sum_{w \in V_{1}}\left(\tau_{w}^{\prime}+\tau_{w}^{\prime \prime}\right) w+\sum_{w \in V_{2}}\left(\tau_{w}^{\prime}+\tau_{w}^{\prime \prime}-1\right) w+\sum_{w \in V_{2}} w .
$$

Alternatively, this means $\alpha^{1}=\alpha^{\prime}+\sum_{w \in V_{2}} w \in b(u \mid X)$. Similarly, we derive from the second relation in $(6.20)$ that

$$
\alpha^{2}=\alpha^{\prime}+\sum_{w \in V_{2}^{\prime}} w \in b(-u \mid X)
$$


holds for some $V_{2}^{\prime} \subseteq W(X)$ (where $V_{2}^{\prime}$ is possibly different from $V_{2}$ ). Now, note that $W(X) \subseteq Y$ so that $\alpha^{\prime} \in Z_{j}$ implies $\alpha^{1} \in Z_{j}$. Thus set

$$
\alpha=\alpha^{1}=\alpha^{\prime}+\sum_{w \in V_{2}} w
$$

so that

$$
\alpha^{2}=\alpha^{\prime}+\sum_{w \in V_{2}^{\prime}} w=\alpha+\left(\sum_{w \in V_{2}^{\prime}} w-\sum_{w \in V_{2}} w\right) \in b(-u \mid X) .
$$

Hence (6.14) follows again from (6.15) as soon as we have shown that $\alpha$ constructed above is different from zero. To see this note that $W(X) \subseteq \partial\langle X\rangle_{+}=\partial\langle\widehat{X}\rangle_{+}$. Since $\alpha=0$ means $\alpha^{\prime}=-\sum_{w \in V_{2}} w$ we get $-\alpha^{\prime} \in \partial\langle\widehat{X}\rangle_{+}$. However, this is contradicted by the fact that by construction $-\alpha^{\prime} \in \operatorname{int} Z(\widehat{X}) \subset \operatorname{int}\langle\widehat{X}\rangle_{+}=\operatorname{int}\langle X\rangle_{+}$. Thus the proof of Lemma 6.3 is complete.

\section{REFERENCES}

1. H. Anand, V. C. Dumir and H. Gupta, A combinatorial distribution problem, Duke Math. J. 33 (1966), 757-769.

2. E. T. Bell, Interpolated denumerants and Lambert series, Amer. J. Math. 65 (1943), 382-386.

3. C. de Boor and K. Höllig, B-splines from parallelepipeds, J. Analyse Math. 42 (1982/83), 99-115.

4. W. Dahmen, On multivariate B-splines, SIAM J. Numer. Anal. 17 (1980), 179-191.

5. W. Dahmen and C. A. Micchelli, Translates of multivariate splines, Linear Algebra and its Applications 52/53 (1983), 217-234.

6. - Recent progress in multivariate splines, Approximation Theory IV (C. K. Chui, L. L. Schumaker, J. D. Ward, eds.), Academic Press, New York, 1983, pp. 27-121.

7. __ On the solution of certain systems of partial difference equations and linear dependence of translates of box splines, Trans. Amer. Math. Soc. 292 (1985), 305-320.

8. _ On the local linear independence of translates of a box spline, IBM Research Report RC 10168 (1983); Studia Math. 82 (1985), 243-263.

9. __ Subdivision algorithms for the generation of box spline surfaces, Computer Aided Geometric Design 1 (1984), 115-129.

10. A. J. Hoffman and J. B. Kruskal, Integral boundary points of convex polyhedra, Ann. of Math. Studies, no. 38, Princeton Univ. Press, Princeton, N.J., 1956, pp. 223-241.

11. P. A. MacMahon, Combinatory analysis, Vols. I, II, Cambridge Univ. Press, 1916, reprinted by Chelsea, New York, 1960.

12. J. Riordan, Introduction to combinatorial analysis, Wiley, New York, 1958.

13. R. P. Stanley, Combinatorics and commutative algebra, Birkhäuser, Boston, Mass., 1983.

14. __ Linear diophantine equations and local cohomology, Invent. Math. 68 (1982), 175-193.

15. D. J. A. Welsh, Matroid theory, Academic Press, New York, 1976.

Fachbereich Mathematik, Freie Universitat Berlin, Arnimallee 2-6, 1000 BERLIN (WEST) 33, GERMANY

Department of Mathematics, iBM T. J. Watson Research Center, Yorktown HEIGHTS, NEW YORK 10598 